\title{
A NEW TEACHING METHOD AIMED AT ELIMINATING THE CAUSES OF STUDENTS' UNSUCCESSFUL ALGORITHMIC PROBLEM SOLVING WITH PARAMETER

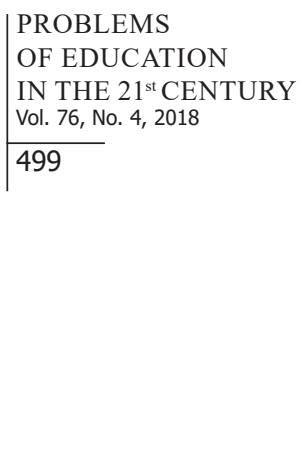

\author{
Dalibor Gonda \\ University of Žilina, Slovakia \\ E-mail: barboragondova@gmail.com
}

Anna Tirpakova

Constantine the Philosopher University in Nitra, Slovakia

E-mail: atirpakova@gmail.com

\begin{abstract}
The new method of teaching the problems with a parameter was designed based on the identification of the reasons for the low success rate of the kids in solving the problems. In order to identify these causes and to find ways of their elimination, pedagogical research was carried out in two phases at selected universities of Slovakia (126 students) in 2016-2017. In the first stage the validity of the research hypothesis was confirmed, that the cause of the kids' poor success in solving problems with the parameter is an algorithmic approach to their solution, a misunderstanding of the concept of a parameter and a formal determination of solvability conditions. Based on this, a new method of teaching the tasks with a parameter was developed. Its main idea is focusing on understanding the term parameter and showing its creative use in solving multiple tasks. In the second stage of the research, the success of the new teaching method was also verified experimentally. The results of the experiment confirmed the success of the students in solving the problems with the parameter, confirming that the basis of the success in solving the problems is the understanding of the term parameter and the ability to find their own solution to the task. It has turned out that the transition from the method of solving equations with a parameter by algorithms to the solution method by understanding the basic concepts and possibilities of their use is one of the methods to improve the teaching of mathematics.
\end{abstract}

Keywords: algorithmic solution, formal conditions, mathematical didactics, problems with the parameter, understanding of the concept.

\section{Introduction}

Teaching and learning can be broadly classified into two types. The names of months, days, physical quantities, names of people, animals, objects etc. were created by agreements. They were not logically derived. It is sufficient to learn them without understanding for their acquisition. Principles, laws, theories arose on the basis of logical operations. It is not enough to learn their wording. For their acquisition they need to be learned with understanding. Mathematical knowledge arose as a result of logical reasoning and operations of solving tasks of practical life. Using mathematical knowledge to solve new challenges requires an understanding of the already acquired solving procedures of already solved tasks. To develop mathematical knowledge and thinking it is needed to seek answers how to teach math so that the students 
Dalibor GONDA, Anna TIRPAKOVA. A new teaching method aimed at eliminating the causes of students' unsuccessful algorithmic problem solving with parameter

IN THE $21^{\text {st }}$ CENTURY Vol. 76, No. 4, 2018

500

can understand and at the same time the root causes of misunderstanding mathematics must be looked for (Sierpinska, 1994; Ma, 1999).

The students seem to have to go to great effort to learn the ways of solving the mathematical tasks without understanding. This attitude to learning mathematics results from the way they learn other, mainly humanity-based subjects. These allegations are based on several studies. However, scientists found that the students who were able to memorize easier, did not belong to the better ones, they did not have particular mathematical abilities not shared by other students (Supekar et al., 2013). Teaching mathematics in the early stages of mathematical education contributes to the students' effort to memorize only solving procedure. Wolfram (2010) points out an inappropriate way of teaching mathematics. He says that $80 \%$ of school mathematics focuses on manual calculations. The primary objective of the mathematical education is to create a quality calculating apparatus - quality mathematical basis.

At this version, teaching mathematics is focused on acquiring the basic terms and procedures for solving the mathematical tasks. The teacher gives students the curriculum mostly in the definitive final form. The teacher's explanation of the procedure is followed by training the solving procedures based on similar tasks, as it was the prime one. The students deal with most tasks by imitating, repeating the solving process that is already familiar for them. In some time, students will get familiar with the idea that to be able to solve the task is identical to calculating algorithms. They (students) pay a little attention to understanding why the calculating algorithm was used for solving the task. Finally, they get the idea that the successful solving of mathematical tasks is based on adopting solving procedures without understanding them. It is enough to match the task assignment with the correct calculating algorithm. But in fact, the successful investigators of mathematical tasks are those students who can already adopt solving procedures in other areas of mathematics. Learning mathematics should contribute to developing a more in-depth learning style. It lies in trying to understand the curriculum, capture its meaning, and involve acquired knowledge into existing knowledge structures. Students learn not only to meet the requirements, but they want to apply the acquired knowledge in practice (Kubiatko, Torkar \& Rovnanova 2017). However, there is a necessity of teaching and learning with understanding that is essential for success in mathematics. Teaching and learning with understanding requires a different approach of teachers and learners. Novelty lies primarily in the fact that there is a priority to focus on as to why the problem is solved in this way (not how it is solved), so the logical procedures of learning are used, and logical chains are created (Petty,2014).

\section{From the Calculating Algorithms to the Methods of Solving}

The development of mathematical thinking can be divided into two stages. The first, essential step is the acquisition of elementary calculating algorithms. The term algorithm means a set of clearly defined rules governing the succession of implementing a finite number of elementary operations that ensures each task of that type will be solved in finite time. The algorithm can be characterized by its basic characteristics: rithm).

1. Elementarism: a finite number of simple, easily achievable actions (the steps of algo-

2. Determinism: After each step, it can be said that the algorithm has already ended and if not, what step should follow.

3. Terminality: The process described by an algorithm will end after some final time.

4. Finiteness: an algorithm leads the calculation from the input data to the result.

5. Mass scale: an algorithm is defined to solve the big group of tasks of the same type. (Znám, 1986)

In mathematics the basic calculating algorithms are considered to be all the calculating operations with expressions and solving algebraic equations, inequalities and their sets. On 
the basis of the teacher's explanation, the students acquire calculating algorithms and practice them on the appropriate number of tasks. It is possible to talk about math "drill", without which it is impossible to be a successful solver of mathematical problems. Basically, the students are expected to automate the implementation of the basic calculating algorithms. At this stage information-receptive didactic method in combination with the reproductive method are used (Patlak, 2004). It is very important that repeated use of basic calculating algorithms will help the student to receive the necessary skill in their use. The teacher can, even at this stage, hope to achieve that students acquire these calculating algorithms at least at the level of understanding, not only at the level of memorization.

The second stage of mathematical education is teaching algorithmic rules. Algorithmic guidelines, in contrast to an algorithm, are not characterized by determination or formality. Turek (2008) stated that the individual operations take the content character and require mental activities, based on the understanding of their meaning. Therefore, it is necessary to put some stress on teaching them. At this stage, it is very important to monitor and control the understanding of supporting ideas of the method. It is recommended to choose the tasks to solve them using the algorithmic guidelines applied in different variations. This prevents students from memorizing the algorithmic guidelines as the calculating algorithm. Solving tasks by using algorithmic guidelines leads to application of acquired algorithms in different areas of mathematics and other disciplines, or in practical everyday life. At this stage, the mathematical "drill" is substituted by mathematical reasoning. This is the stage in which students learn to create the solving of the task. The essential feature of this phase of mathematical learning is to develop capabilities to deal with the task by applying already acquired knowledge and skills from different areas, not just mathematics. In addition to new knowledge there is the place to apply already acquired skills. A student learns that the first step of task solving is not to count but to think. A priority for him/her is to create a solution and not to match task assignment with a calculating algorithm. In principle, there is a fundamental shift in thinking and approaching to dealing with (not only) mathematical problems. Shift is from "repeating" of what I learned to "wonder" how to use what I know. Therefore, it is necessary to remember each step of the algorithmic guidelines, but at the same time, it is not a sufficient condition for using the algorithmic guidelines in solving problems. The teacher becomes a moderator of solving. A didactic heuristic method is considered the most appropriate.

In terms of successful solving of mathematical problems, transition from the acquisition of basic calculating algorithms to the stage of algorithmic guidelines becomes critical. In most cases, the problem lies in students trying to understand the discussed methods of solving algorithms which are enough to learn and match them with the task assignment. In this context, a serious problem appears to be the indirect methods of solving mathematical problems as the most frequently used algorithmic guidelines (Kramarski, Mevarech \& Arami, 2002).

Even these methods have their internal structure, a certain sequence of steps. The perception of calculation algorithms is very restrictive for the further development of mathematical thinking of learners. When using the indirect methods, the task often needs to be divided into the sub-tasks that can already be solved by the relevant calculating algorithms. Indirect methods are largely universal methods and they can help to solve different types of problems. Their effective use is necessary, in addition to acquiring basic structure of the method, to understand the method. Given the increased level of acquiring algorithmic guidelines, there is a difference in the successful tasks being solved by individual students (Turek, 2008).

The tasks with parameters create a large group of tasks solved by indirect methods. The task that contains the parameter is a set of the same type of tasks. The particular task is obtained by replacing the parameter by a number. If a parameter is put to the task, the task does not change (quadratic equation remains quadratic). The parameter will be added as an unknown and new term to solve the equation with similar content.

\author{
PROBLEMS \\ OF EDUCATION \\ IN THE $21^{\text {st }}$ CENTURY \\ Vol. 76, No. 4, 2018 \\ 501
}


Dalibor GONDA, Anna TIRPAKOVA. A new teaching method aimed at eliminating the causes of students' unsuccessful algorithmic problem solving with parameter

IN THE $21^{\text {st }}$ CENTURY Vol. 76, No. 4, 2018

502

Based on research dealing with how students understand the term of a variable (MacGregor \& Stacey, 1993; Bednarz \& Lee, 1996; Trigueros \& Ursini, 1999; Bardini, Radford \& Sabena, 2005), the similar problems with the understanding of the term parameter are expected. "These studies show that the variable is often viewed as a "potentially determined" number. The students see it as a temporarily unknown number that will be determined at some point. There are probably the roots of confusing the term of a variable with the term of unknown. The term of unknown means the unknown number that is determined when solving equations or inequalities (Schoenfeld \& Arcavi; 1988, Radford, 1996). It is supposed that the reasons for a problem to solve tasks with parameter are related to students' access to learning mathematics. According to the current mathematics education the teaching of mathematics is often based on the transmission of ready knowledge and their memorization, while it should be based on the creative learning process with the active participation of learners (Navarro-Ibarra 2017). This system teaches students to match a learned process and an algorithm, with the assigned task. Therefore, the task with parameter is perceived as a new type of task, different from an analogous task with no parameter. The teacher is expected to teach them the new algorithm for finding a solution. But the presence of a parameter does not change the strategy of calculating, it requires dividing the task into individual parts - to atomize. Atomization is the result of thinking when looking for solutions. It is not an automatic step of algorithm. The main cause of little success in solving the tasks with parameter is the students' effort to deal with the task using algorithms and matching calculations with the assigned task, without sufficient understanding of each step of the solution.

Based on the above-mentioned considerations, the main hypothesis was established that the main reason of a low success rate in solving tasks with parameter is probably the students' effort to solve all mathematical tasks algorithmically, i.e. by matching the learned algorithm to the task assignment without understanding individual steps of the solution. To verify this hypothesis, the pedagogical research in selected universities in Slovakia in 2016-2017was conducted. For the analysis of the research results, selected statistical methods were used.

\section{Methodology of Research}

Pedagogical research was carried out in 2016-2017 at three pedagogical faculties of the selected universities in Slovakia. Pedagogical research was carried out in two stages. In the first stage of research, our goal was to identify the causes of students' failure to solve problems with the parameter. The next step was, based on this identification, to propose a suitable method for the removal of the problems, it means to propose a new teaching method that could eliminate the identified causes of students' failure to solve problems with the parameter.

In the second stage of pedagogical research, the effectiveness and efficiency of the proposed new method to eliminate the above-mentioned causes were experimentally verified.

In both stages of the research, the research sample consisted of 126 first-year students who have been trained for the profession of a teacher of mathematics. The tasks with a parameter are considered to be more challenging in the technical literature. That's why the students were deliberately chosen, who, after graduating from secondary school, chose to study mathematics. With the chosen research sample, the ability to solve problems with the parameter at an appropriate level could be expected.

The students were randomly divided into three groups - an experimental group (EG) and two control groups (CG1) and CG2). The experimental group was formed by 27 students, the CG1 control group included 56 students and there were 43 students in the CG2 group. After placing the students into individual groups, i.e. before the beginning of the experiment, it was necessary to verify the equivalence of all three groups. In order to assess the equivalence of the groups, the same mathematical test (pre-test), consisting of four tasks, was given to them at the 
Dalibor GONDA, Anna TIRPAKOVA. A new teaching method aimed at eliminating the causes of students' unsuccessful algorithmic problem solving with parameter

very beginning of the experiment. The first two tasks were represented by common inequalities without parameters. They were deliberately included in the pre-test to demonstrate the ability of the respondents to solve basic types of inequalities. Two other tasks contained similar types of inequalities. As they were crucial for the pilot research, they had to be chosen very carefully. Our aim was to identify the causes of a low students' success rate in solving the tasks with parameters, and also verify research hypothesis that the main cause of this low success rate is the effort of students to solve all mathematical tasks algorithmically. For the sake of completeness, not only the key tasks ( 3 and 4 ) in the pre-test but also a brief outline of their solutions.

Task 1: Solve the inequality with parameter $a:|x|<\frac{a}{x}$.

Solution. From the instructions it is evident that if $x \neq 0$ and the given inequality has no solution.

First method. Task 1 is solved as an absolute value inequality in the following way:

For $x>0$ the inequality is solved $x<\frac{a}{x}$.

Given that the unknown $x$ acquires only positive values, after the removal of the fraction from the inequality, the simplified $x^{2}<a$ was solved. Obviously, this inequality has solutions only for the positive values of a parameter $a$. Its solution on the set of positive numbers is $x \in$ $(0 ; \sqrt{a})$. For the inequality was solved

$$
-x<\frac{a}{x}
$$

Given that the unknown $x$ acquires only negative values, after removing the fraction from the inequality, the simplified $x^{2}<-a$ inequality was solved. Obviously, this inequality has solutions only for the negative values of a parameter $a$. Its solution in a set of negative numbers is $x \in(0 ;-\sqrt{ }-a)$. The obtained solution is supposed to be written in the table.

Second method. Task 1 is solved as an inequality with an unknown parameter in the denominator. The right side is canceled and a fraction on the left one was created. After these adjustments, there is an inequality:

$$
\frac{a-x|x|}{x}>0 .
$$

In $x=\mathrm{o}$ the denominator vanishes. The zeros of the numerator are obtained by solving the equation

$$
a-x|x|=0 .
$$

For $x>0$ the equation $a-x^{2}=0$ is solved. Solution of this equation is $x= \pm \sqrt{a}$ for the positive values of parameter $a$. Solutions of the tasks are clearly written in the table (Table 1), knowing that only positive values of the variable should be written there.

Table 1. Solution of Task 1 (Second method for positive values of the variable ).

\begin{tabular}{ccc}
\hline & $(0 ; \sqrt{ } a)$ & $(\sqrt{ } a ; \infty)$ \\
\hline$a-x|x|$ & + & - \\
\hline$x$ & + & + \\
\hline$\frac{a-x|x|}{x}$ & + & - \\
\hline
\end{tabular}
$\in(0 ; \sqrt{a})$.

Based on the results listed in the table (Table 2) for the parameter $a>0$ the solution is $x$

For $x<0$, an equation $a+x^{2}=0$ is solved. Solution of this equation is $x= \pm \sqrt{ }-a$ for nega- 
Dalibor GONDA, Anna TIRPAKOVA. A new teaching method aimed at eliminating the causes of students' unsuccessful algorithmic problem solving with parameter

OF EDUCATIOI

IN THE $21^{\text {st }}$ CENTURY Vol. 76, No. 4, 2018

504

tive values of the parameter $a$. The solutions for the negative values of the variable $x$ are written clearly in the following table (Table 2).

Table 2. Solutions of Task 1 (Second method for negative values of the variable).

\begin{tabular}{ccc}
\hline & $(-\infty ;-\sqrt{-a})$ & $(-\sqrt{-a ; 0})$ \\
\hline$a-x|x|$ & + & - \\
\hline$x$ & - & - \\
\hline$\frac{a-x|x|}{x}$ & - & + \\
\hline
\end{tabular}

Based on the results listed in the table (Table 2) for the parameter $a<0$ solution of the inequality is $x \in(-\sqrt{-} a ; 0)$.

Task 2: Solve the quadratic inequality with the parameter $a: x^{2}+a x>0$.

\section{Solution.}

First method. The task as a quadratic inequality with parameter is solved. First the value of the discriminant on which the number of solutions of the corresponding quadratic equation depends is determined.

$$
x^{2}+a x=0
$$

In this case, the discriminant is equal to an expression $D=a^{2}$.

If the expression $a^{2}$ is positive $(D>0)$, the corresponding quadratic equation (2.1) has two different real roots. So, it can be written down as:

$$
a^{2}>0 \Rightarrow a \in R-\{0\} \Rightarrow x_{1,2}=0 ;-a .
$$

In the next step, the quadratic inequality $x^{2}+a x>0$ with the parameter $a \in R-\{0\}$ is solved. An inequality is solved by the method of zero points. By using this method, there will be roots of a corresponding quadratic equation $x_{1}=0, x_{2}=-a$. Based on this zero-point method, the domain of the expression $x^{2}+a x$ into intervals is divided, on which this expression acquires the same "sign" values (positive or negative). For $a>0$ a zero point is negative: $x=-a$. The solutions will be written down in the following table (Table 3).

Table 3. Solutions of task 4 for $a>0$ (First method).

\begin{tabular}{cccc}
\hline & $(-\infty ;-a)$ & $(-a ; 0)$ & $(0 ; \infty)$ \\
\hline$x$ & - & - & + \\
\hline$x+a$ & - & + & + \\
\hline$x(x+a)$ & + & - & + \\
\hline
\end{tabular}

Based on the solutions listed in the table (Table 3 ) for $a>0$ the solution of inequality is $x \in(-\infty ;-a) \cup(0 ; \infty)$. 
Dalibor GONDA, Anna TIRPAKOVA. A new teaching method aimed at eliminating the causes of students' unsuccessful algorithmic problem solving with parameter

For $a<0$ a zero point $x=-a$ is positive. The solutions will be written down in the folVol. 76, No. 4, 2018 lowing table (Table 4).

Table 4. Solutions of task 4 for $a<0$ (First method).

\begin{tabular}{cccc}
\hline & $(-\infty ; 0)$ & $(0 ;-a)$ & $(-a ; \infty)$ \\
\hline$x$ & - & + & + \\
\hline$x+a$ & - & - & + \\
\hline$x(x+a)$ & + & - & + \\
\hline
\end{tabular}

Based on the solutions listed in the table (Table 4) for $a<0$ the solution of inequality is $x \in(-\infty ; 0) \cup(-a ; \infty)$.

If the expression $a^{2}$ equals zero $(D=0)$, the corresponding quadratic equation has the only solution $x_{p}=0$. The solution to the quadratic inequality is in this case $x \in R-\{0\}$. As the expression $a^{2}$ does not acquire negative values, the case of a negative discriminant is not taken into consideration.

At the end the solution of the inequality (Task 2) is clearly written in the Table 5.

Table 5. Solution of Task 2.

\begin{tabular}{cc}
\hline$a$ & $x$ \\
\hline 0 & $R-\{0\}$ \\
\hline$a>0$ & $(-\infty ; a) \cup(0 ; \infty)$ \\
\hline$a<0$ & $(-\infty ; 0) \cup(-a ; \infty)$ \\
\hline
\end{tabular}

Second method. Task 4 is solved as a quadratic inequality without an absolute member. The zero-point method is used. The expression on the left side of the inequality was modified into the product form. There is an inequality in the form:

$$
x(x+a)>0
$$

The expression on the left side acquires the value zero for $x=0$ and $x=-a$. On the basis of these zero points, the domain of the expression $x^{2}+a x$ was divided into the intervals on which this expression acquires the same "sign" values (positive or negative) For $a>0$ a zero point $x=-a$ is negative. A solution table is created (Table 6).

Table 6. Solution of Task 2 for $a>0$ (Second method).

\begin{tabular}{cccc}
\hline & $(-\infty ;-a)$ & $(-a ; 0)$ & $(0 ; \infty)$ \\
\hline$x$ & - & - & + \\
\hline$x+a$ & - & + & + \\
\hline$x(x+a)$ & + & - & + \\
\hline
\end{tabular}

Based on the results listed in the table (Table 6 ) for $a>0$ the solution of the inequality is $x \in(-\infty ;-a) \cup(0 ; \infty)$. For $a<0$ the zero point $x=-a$ is positive. A solution table is created (Table 7). 
Dalibor GONDA, Anna TIRPAKOVA. A new teaching method aimed at eliminating the causes of students' unsuccessful algorithmic problem solving with parameter

PROBLEMS

OF EDUCATION

IN THE $21^{\text {st }}$ CENTURY

Vol. 76, No. 4, 2018

506

Table 7. Solution of Task 2 for $a<0$ (Second method).

\begin{tabular}{cccc}
\hline & $(-\infty ; 0)$ & $(0 ;-a)$ & $(-a ; \infty)$ \\
\hline$x$ & - & + & + \\
\hline$x+a$ & - & - & + \\
\hline$x(x+a)$ & + & - & + \\
\hline
\end{tabular}

Based on the results listed in the table (Table 7) for $a<0$ the solution of the inequality is $x \in(-\infty ; 0) \cup(-a ; \infty)$. For $a=0$ there is only one zero point $x=0$. In this case the solution of the inequality is $x \in R-\{0\}$.

From the stated solution of both inequalities, the possibility of perceiving the tasks can be seen from various viewpoints. This fact has extended the range of options for choosing solutions. In both tasks, however, the respondents had to deal with the key element of the solution, with the expression $-a$.

First Stage of Pedagogical Research: Identification of Causes of Failure of Students to Solve Problems with a Parameter

As it has already been mentioned, in the first stage of our research, our goal was to identify the causes of students' failure to solve problems with the parameter. It was proceeded as follows. First, the results that the students of each group achieved in the pre-test were evaluated.

Through a detailed analysis of the pre-test results obtained by the students of all three groups (EG, CG1 and CG2), the hypothesis was verified that the main cause of a low success rate in solving tasks with parameter is the students' effort to solve all mathematical tasks "algorithmically" i. e. by matching the calculation to the instructions of the task without sufficient understanding of the individual steps of the solution.

Firstly, by analyzing the solutions of the assigned tasks it was found out that the most frequent deficiency related to the algorithmic approach of our respondents was the perception of the term " $-a$ ". Many of the students considered the term to be always negative and adequately they considered the term " $a$ " to be always positive. This kind of perception is the result of student's "algorithmization" of elimination of absolute value from an equation or inequality. Instead of understanding the term of an absolute value, students very often acquire the following short algorithm: "For a positive value of the expression in an absolute value, the absolute value can be omitted and for the negative value of the expression in an absolute value I will write it without an absolute value in the next step and change the sign of each member to the opposite one." In such an algorithmic approach, a sign before an expression decides whether it is positive or negative, regardless of the fact which group of numbers a variable or parameter represents. The most task solutions in the pre-test of all three groups showed that this algorithmic misconception is the main cause of an improper solution of tasks.

The second major problem that was encountered when solving the pre-test tasks is represented by so called "formal conditions". Based on the respondents' response analysis, the perception of the conditions can be summarized as follows: defining the conditions for them is the task that needs to be done under certain circumstances arising from the task assignment. Also, in this case it is matching some algorithm to the task instructions. To a great extent, it can be said that students set conditions because they remember their determination as one of the solution steps. During the calculation process they seldom work with them, they do not use them to make the calculation part of the task more efficient. Tasks with parameter show an effort to determine conditions equally based on memory. And the formality of determining the 
conditions (determining the conditions to meet the rule of necessity of their determination) is manifestation of misunderstanding of their function in the task solution. The tasks with parameter require active work with conditions for either a parameter or a variable.

Finally, it was assumed that by analyzing the results of the pre-test there have been identified two serious causes of a low success rate in solving tasks with parameter, namely perception of the negative expression and the problem when determining the conditions for the task solution. Thus, it can be stated that a detailed analysis of the pre-test results written by students in all three groups (EG, CG1 and CG2) confirmed our hypothesis that the main cause of a low success rate in solving tasks with parameter is the effort of students to solve all mathematical tasks "algorithmically", i.e. by matching the calculation to the task assignment without sufficient understanding of the individual steps of the solution. Based on the shortcomings that have been identified, a new method for their removal has been proposed.

\section{A New Teaching Method Aimed at Increasing the Success of the Problem Solving with the Parameter}

The next step of the first stage of the pedagogical research was to design a suitable method to avoid problems with task solving with a parameter, i.e. to propose a new teaching method that could eliminate the causes of students' failure to solve problems with the parameter.

In the pre-test, there have been identified two serious shortcomings, which have a common denominator: a misunderstanding of the term parameter by students. The parameter was not perceived as a representative of the known set of numbers but as another variable in the task. This misunderstanding in connection with the algorithmic approach to task solving has led not only to incorrect results but also to the wrong process of searching a result. To eliminate the shortcomings, a method has been developed aimed at the introduction of the term parameter, whose main idea and methodology are briefly outlined in the following tasks.

Before introducing the term parameter, there was a discussion with students on the subject: "What role does a sign before the unknown variable have?" As the basis of the discussion, the definition of an absolute value was used. In the first phase of the discussion, the fact was considered that students learn to distinguish between the numerical expression -3 and the expression $-a$ essential. It is important to point out that -3 represents a negative number but $-a$ has to be perceived as the opposite expression to the expression $a$. At the same time, -3 is also an opposite number to 3. Symbol "-" can be interpreted in the stated context in two meanings: 1. a negative one, 2. an opposite one. Both meanings were introduced to students during presenting and teaching the topic: Whole numbers. Because of the fact that they are taught that the opposite number to the number -3 is number $3(-(-3))$ is not used, in the minds of students only the link: symbol - (minus) before the number is equal to the term "negative" is kept. On the basis of this concept they analogically perceive minus before a variable as the negative value of the variable. Therefore, when teaching a thematic unit of Equations with an absolute value, the emphasis should be placed on equality: minus = "opposite". For this reason, if the expression in an absolute value on a given interval is negative, it will be replaced by the opposite expression but without an absolute value.

This fact has been used in the teaching process - when solving inequalities, where the parameter as an effective means of carrying out the checking of answers was introduced. The perception of the parameter as a representative of the chosen set of numbers was emphasized. At the same time, the students were unequivocally made to distinguish between the notion of a parameter and the notion of an unknown variable. The proposed procedure, where students' attention was clearly drawn to the above-mentioned differences, is illustrated in the following task. A simple task, whose solution does not make any problems for students, has deliberately been chosen. 
Dalibor GONDA, Anna TIRPAKOVA. A new teaching method aimed at eliminating the causes of students' unsuccessful algorithmic problem solving with parameter

\section{PROBLEMS \\ OF EDUCATION \\ IN THE $21^{\text {st }}$ CENTURY Vol. 76, No. 4, 2018 \\ 508}

Task 3. In the set $\mathrm{R}$ solve inequality $2 x-3>x+6$.

Solution. After simple adjustments, an inequality $x>9$ is obtained. A set of solutions for a given inequality is written using the interval $K=(9 ; \infty)$.

The task is solved, but the students need to be shown that such solution is incomplete. There has arisen a situation where it is useful to introduce the term parameter. It can be justified as follows: the students were asked to test the correctness of the solution - i.e. to check their answers. At the same time, it must be emphasized that even in the case of inequalities it is necessary to be able to verify the correctness of the task solution - even if the test is not a necessary part of the solution. Numbers that match the specified inequality are written in two ways: in the form of inequality $x>9$ or in the form of set $K=(9 ; \infty)$. In both cases, it is a mathematical enrollment of all real numbers greater than 9. If the analogy is used with test of correctness when equations are being solved, all real numbers larger than 9 should sequentially be placed. The problem is that there are an infinite number of them. The students can be reminded that in the test of correctness, we cannot place either inequality or a set; we can only place a number. Therefore, we need to find a new way of writing all the numbers larger than 9 , and we need it to be a "number". Numbers greater than 9 can be written as follows: $9+1,9+2,9+3, \ldots$ Any number greater than 9 can be written by adding an (any) positive number to the number 9 . In mathematics, for writing any number we can use, for example, the letter to which we attach the set of numbers it represents. Therefore, the entry we are looking for can be, e.g.: $9+a ; a \in(0 ; \infty)$. In our case, we used a "letter" to write numbers with the same property, and this "letter" gets the name parameter. In general, in mathematics a parameter is used in case when we want to write multiple objects with the same property by using one notation (e.g. expression, equation, etc.) Notation $9+\mathrm{a}$ is the set of numbers that we can place into an inequality. After placing these numbers, we do just one test for correctness for infinitely many solutions of inequality. We point out that the set of numbers which was added to the notation $9+\mathrm{a}$ can be also perceived as the condition that the parameter must meet for our expression to represent the required set of numbers. In this way, students have "created" a connection between the expression containing the parameter and the condition that the parameter must meet for the expression to have the required properties. The described way of introducing the term parameter has been revised with students by solving similar suitable tasks. The emphasis was put also on the work with conditions - with respect to parameter.

The new method of teaching the term parameter will be illustrated in the following example. The example was chosen intentionally - when solving it, it is necessary to understand the term parameter as well as to work actively with conditions.

Task 4. For what values of the parameter $a \in R$ is every $x \in R$ solution of inequality

$$
\frac{x^{2}-8 x+20}{a x^{2}+2(a+1) x+9 a+4}<0
$$

Solution. Students k1now how to work with the method of zero points. Its algorithmic use leads them to finding out that an expression in the denominator has no zero points (equation $x^{2}-8 x+20=0$ has not solution in the set $R$ ). The zero points of the expression in the denominator are dependent on the parameter and their indication on the numerical axis is very problematic. At this point of the task solution, students can see a serious lack of algorithmic approach. It does not always solve the task, or the solution is too demanding and lengthy (Turek 2008). If the students acquired the term at a sufficient level, they can find their own solving procedure. To solve the task, we will stop using algorithm and start forming our own solution based on our own knowledge. With students, we will discuss the meaning of a zero point. Based on the understanding of this term, they will deduce that the expression which has no zero points acquires the resulting values with the same sign. On the basis of the sign before an absolute member, we will determine that the expression $x^{2}-8 x+20$ acquires positive values for all $x \in R$. 
The expression on the left side of the specified inequality is a fraction. If it is to be negative, the numerator and the denominator must have different resulting signs. The expression in the numerator is for all $x \in R$. positive, therefore the expression $a x^{2}+2(a+1) x+9 a+4$ has to be "always" negative. The fulfillment of this fact is realized by the following consideration: Through the parameter we first ensure that the expression has a constant resulting sign. As the next step of the solution we use the zero-point function. If the expression is supposed to have the same final sign for all the possible unknown values $x$, then it can't have zero points (except "useless" zero points). That means that a quadratic equation

$$
a x^{2}+2(a+1) x+9 a+4=0
$$

cannot have a solution in the set $\mathrm{R}$. This condition is fulfilled if the discriminant of the equation (2) is negative. This is the way how we got an inequality

$$
4(a+1) 2-4 a(9 a+4)<0 .
$$

Solution of this inequality, which we after the adjustments solve by the method of zero points, is set $K_{1}=\left(-\infty ;-\frac{1}{2}\right) \cup(1 ; \infty)$.

In the next step, the students are explained how to ensure with the help of the parameter $a$ that the resulting sign of the expression is negative. First, students need to be emphasized that the denominator of a fraction in a given inequality $a x^{2}+2(a+1) x+9 a+4$ is a quadratic triple with the parameter which in case that $a \in K_{1}$ has a constant resulting sign for all $x \in R$. From the zero-point method we know that after any number is placed for variable $x$ to the given triplet, we will find out a resulting sign for all $x \in R$. We also know that after we place a zero in the quadratic triplet, the resulting triplet sign is identical with the sign before an absolute member. In our case an absolute member is an expression $9 a+4$. That is why we are solving an inequality

$$
9 a+4<0 .
$$

Solution of this inequality is $K_{2}=\left(-\infty ;-\frac{4}{9}\right)$. Based on previous considerations, the parameter must meet both conditions. Therefore, all parameter values match the task $a \in K_{1}$ $\cap K_{2}=\left(-\infty ;-\frac{1}{2}\right)$.

On the basis of the two tasks we have methodically described the proposed method which is aimed at eliminating the causes of failure, respectively the method aimed at improving the success rate when solving inequalities with parameter. This new method of teaching selected parts of mathematics was subsequently and experimentally applied in the teaching process in EG.

\section{Second Stage of the Pedagogical Research: Verification of the Effectiveness of the New Method}

Before we verify the effectiveness of the new proposed method to eliminate the reasons for students' failure to solve problems with the parameter, the subject of the pedagogical research was also to verify the equivalence of the selected three groups of students - EG, CG1 and CG2. The equivalence of the groups was verified by comparing the results that the students of the individual groups achieved in the pre-test. We compared the results using the selected statistical methods described in the following.

As already mentioned, the pre-test included 4 tasks and was the same for all three groups (EG, CG1 and CG2). We evaluated the results of the assigned tasks in the pre-test as follows. 
Dalibor GONDA, Anna TIRPAKOVA. A new teaching method aimed at eliminating the causes of students' unsuccessful algorithmic problem solving with parameter

OF EDUCAT

IN THE $21^{\text {st }}$ CENTURY Vol. 76, No. 4, 2018

Pre-test students for each task could receive from 0 to 4 points, the student could get a maximum of 16 points for the correct solution of all four tasks in the pre-test. The results - the average number of points for each example - obtained by the students in each group in the pre-test are clearly written down in the following table (Table 8). In Table 9, the average success rate of each example in the pre-test in each group is calculated, expressed as a percentage.

Table 8. Results of the pre-test (average numbers of points).

\begin{tabular}{|c|c|c|c|c|}
\hline \multirow[b]{2}{*}{ Groups } & \multicolumn{4}{|c|}{ Example } \\
\hline & 1 & 2 & 3 & 4 \\
\hline CG1 & 3.74 & 4.00 & 0.96 & 1.93 \\
\hline CG2 & 3.98 & 4.00 & 0.98 & 1.66 \\
\hline EXG & 3.93 & 4.00 & 0.96 & 1.57 \\
\hline
\end{tabular}

Table 9. Results of the pre-test (average success rate in percentage).

\begin{tabular}{lcccc}
\hline \multirow{2}{*}{ Groups } & \multicolumn{5}{c}{ Example } \\
\cline { 2 - 5 } & 1 & 2 & 3 & 4 \\
\hline CG1 & 93.5 & 100.0 & 24.1 & 48.1 \\
\hline CG2 & 97.0 & 97.6 & 23.8 & 40.5 \\
\hline EXG & 98.2 & 100.0 & 24.1 & 39.3 \\
\hline
\end{tabular}

Based on the results of the pre-test (Table 8 and Table 9), we can see that there are small differences in the number of average points between the selected three groups of students. We were concerned whether these differences in the results of the pre-test are also statistically significant. Since the assumption of normal distribution of the observed signs is not warranted, we have chosen the Kruskal-Wallis nonparametric method to verify the statistical significance of the differences between the students' groups in the pre-test results. It allows us to test the hypothesis $H_{0}$ that $k(k \geq 3)$ of independent sets comes from the same distribution. Test criteria of the Kruskal-Wallis test is statistics

$$
H=\frac{12}{n(n+1)} \sum_{i=1}^{k} \frac{T_{i}^{2}}{n_{i}}-3(n+1)
$$

which has in case of validity of a zero hypothesis $H_{0}$ asymptotically $\chi^{2}$-distribution with $k-1$ degrees of freedom. The test hypothesis $H_{0}$ is rejected on the significance level $\alpha$ if $H \geq \chi_{\alpha}^{2}(k-1)$, where $\chi_{\alpha}^{2}(k-1)$ is a critical value of $\chi^{2}$ - distribution with $k-1$ degrees of freedom. If we reject the test hypothesis $H_{0}$ against an alternative hypothesis $H_{1}$, which means that $k(k \geq 3)$ of independent sets comes from the same distribution, still there remains an unanswered question about which selections vary significantly from one another. In the variance analysis, we use a multiple comparison of the serial averages in the program STATISTICA realized by the Kruskal-Wallis test of multiple comparison by Z-score 
Dalibor GONDA, Anna TIRPAKOVA. A new teaching method aimed at eliminating the causes of students' unsuccessful algorithmic problem solving with parameter

$$
Z=\frac{\left|\bar{R}_{i}-\bar{R}_{j}\right|}{\sqrt{\frac{N(N+1)}{2}\left(\frac{1}{n_{i}}+\frac{1}{n_{j}}\right)}} ; \quad Z \geq z_{\alpha}
$$

PROBLEMS

OF EDUCATION

IN THE $21^{\text {st }}$ CENTURY

Vol. 76, No. 4, 2018

511

As the result, one table shows the $Z$ - score and second one $p$-values.

The Kruskal-Wallis test was used to verify whether the three groups of students (CG1, CG2 and EG) statistically significantly differ in the observed sign - in the number of points obtained in the pre-test. The Kruskal- Wallis test was carried out in the STATISTICA program. After entering the input data in the output assembly of the computer, we will obtain the value of the test criteria $Z$ and the probability value $p$. We have obtained the following results for the observed sign (the resulting mark in the pre-test) using the STATISTICA program: the value of the test criteria $H=0.3504$ and the probability value $p=0.8393$ Since the calculated value of the probability $p$ is greater than 0.05 , the hypothesis $H_{0}$ cannot be rejected and the observed differences are not statistically significant. The test confirmed that the individual groups do not differ significantly from one another in terms of the number of points scored. The situation is also illustrated in Figure 1.

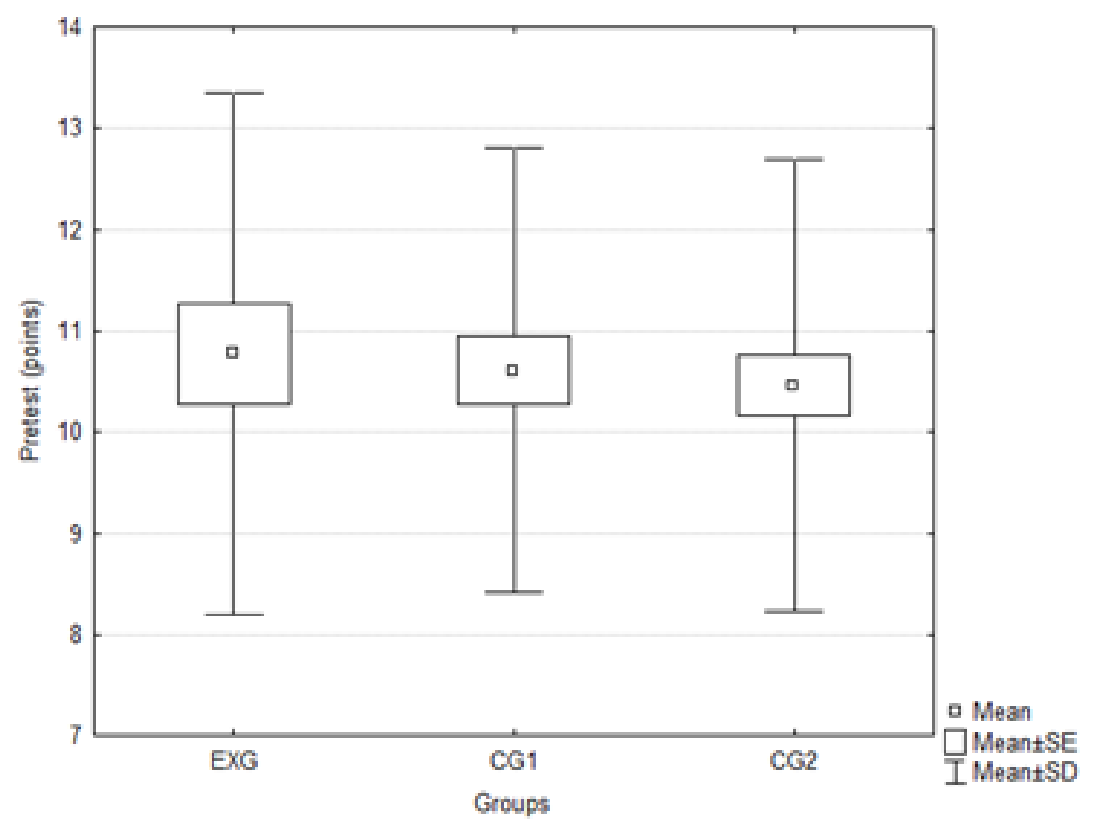

Figure 1. Results of the pre-test (the average number of points in each group).

Based on the above results, all three groups of students were considered as equivalent before starting the experiment.

The aim of the experiment was not only to identify the causes of low success rate when solving the tasks with parameter but also to propose a suitable way to eliminate them, i.e. to propose a new teaching method to eliminate the reasons for failure of students to solve tasks with a parameter. In addition, there was a question if a new method of teaching mathematics, specifically a task with a parameter would help kids to increase their success in solving the given tasks. For this reason, our next goal in the second phase of the research was to verify the 
Dalibor GONDA, Anna TIRPAKOVA. A new teaching method aimed at eliminating the causes of students' unsuccessful algorithmic problem solving with parameter

OF EDUCA

IN THE $21^{\text {st }}$ CENTURY Vol. 76, No. 4, 2018

effectiveness and efficiency of the new teaching method. The effectiveness of the new teaching method aimed at reducing the reasons of failure of the solving of inequalities with a parameter was verified experimentally in three groups of students (CG1, CG2 and EG) frequenting the first year at pedagogical faculties of the selected Slovak universities.

The experiment was being conducted in the educational process for one semester. In CG1 and CG2 groups, the solution of algebraic equations and inequalities with parameter was trained in the traditional way - with the emphasis on the students' understanding of how to handle such tasks. In the EG group, the term parameter was introduced as described above. Subsequently, in all three groups, logarithmic equations and inequalities without parameter were taught. The basic rule for teaching in all three groups was inter alia the fact that the total teaching hours subsidy had to be identical in all of them in order to limit possible changes in the results caused not by the teaching method itself but by the number of teaching hours.

After finishing the experiment, the students of all three groups (EG, CG1 and CG2) wrote the same test - so called post-test. Like the pre-test, also the post-test consisted of four tasks. The first two tasks were once again common inequalities without parameter. Two other tasks were inequalities with parameter of the same type as the first two tasks. Again, here are two tasks listed in the post-test - 5 and 6 - assignment and a brief description of their solution.

Task 5: In the set $R$ solve an inequality with parameter: $\frac{x-3}{a}>10$.

Solution: Given inequality is solved for parameter values . The "letter" in the inequality is not an unknown variable but a parameter. Therefore, it is not an inequality with the unknown variable in the denominator and the expected solution is to remove the fraction from the inequality: If $a \in(0 ; \infty)$, then $x-3>10 a$. After adjustments $x>10 a+3$.

If $a \in(-\infty ; 0)$ then $x-3<10 a$. After adjustments $x<10 a+3$.

Task 6: In the set solve logarithmic inequality with parameter $a: \log \left(\frac{x-2}{a x}\right)>1$. 1. Algorithmic procedure:

Based on the basic sentence on logarithms, the inequality can be solved

$$
\frac{x-2}{a x}>10 .
$$

Subsequent to canceling the right side and adjusting the left side to one fraction, the inequality was solved

$$
\frac{x(1-10 a)-2}{a x}>0 .
$$

It was not stated how to continue with the task solution as the students did not know how to continue.

\section{Non-algorithmic (creative) procedure:}

One of the sentences about logarithms was used and the inequality was solved

$$
\log (\mathrm{x}-2)-\log a x>1 .
$$

The domain of the inequality was defined. Based on the definition of logarithmic functions, it must be

$$
x-2>0 \wedge a x>0 .
$$

From the first inequality results: $x \in(2 ; \infty)$. Then it is used that $x$ is positive and therefore (from the second inequality) only positive values of parameter are possible.

After a few adjustments, we get the inequality 
Dalibor GONDA, Anna TIRPAKOVA. A new teaching method aimed at eliminating the causes of students' unsuccessful algorithmic problem solving with parameter

$$
\log (x-2)>\log (10 a x)
$$

and then the inequality was solved

$$
(x-2)>10 a x
$$

and after adjustments

$$
x(1-10 a)>2 .
$$

In the following solution, the following cases of the parameter were considered:
1) $a>\frac{1}{10}$
$x<\frac{2}{1-10 a}$
2) $a=\frac{1}{10}$
3) $0<a<\frac{1}{10}$
$x>\frac{2}{1-10 a}$

When determining the result of a task, it is still necessary to take into account the conditions for unknown $x \in(2 ; \infty)$. For a parameter the expres- $a>\frac{1}{10}$, sion $1-10 a$ is always negative, and therefore 2 is always negative. Therefore, the non-equilibrium system

$$
2<x<\frac{2}{1-10 a}
$$

does not have a solution for parameter values $a>\frac{1}{10}$.

For a parameter $0<a<\frac{1}{10}$, the expression $1-10 a$ takes the interval values $(0 ; 1)$ and the

expression $\frac{2}{1-10 a}$ is always greater than 2 for the given parameter values. Based on the mentioned above the total solution of the given inequality is $x \in\left(\frac{2}{1-10 a} ; \infty\right) ; a \in\left(0 ; \frac{1}{10}\right)$

\section{Results of Research}

The results - the average number of points (for each task) obtained by the students of each group in the post-test is clearly written down in the following table (Table 10).

Table 10. Results of post-test (average number of points).

\begin{tabular}{lcccc}
\hline \multirow{2}{*}{ Groups } & \multicolumn{5}{c}{ Task } \\
\cline { 2 - 5 } & 1 & 2 & 3 & 4 \\
\hline CG1 & 3.88 & 3.22 & 2.41 & 1.80 \\
\hline CG2 & 3.91 & 3.13 & 2.13 & 1.75 \\
\hline EG & 3.85 & 3.37 & 2.93 & 3.19 \\
\hline
\end{tabular}

In Table 10 it can be seen that among the three groups of students there are differences in the number of average points obtained in the post-test. There was a question arisen whether these differences in the post-test results are also statistically significant. Even in this case, the zero hypothesis $H_{0}$ : was tested that the results achieved in the post-test are the same in all three 
Dalibor GONDA, Anna TIRPAKOVA. A new teaching method aimed at eliminating the causes of students' unsuccessful algorithmic problem solving with parameter

PROBLEMS

OF EDUCATION

IN THE $21^{\text {st }}$ CENTURY

Vol. 76, No. 4, 2018

groups against to the alternative hypothesis $H_{1}$ : that the results achieved in the post-test in all three groups are not the same. Using the Kruskal-Wallis test, the value of the test criteria $H=$ 23.8515 and value of probability ( $\mathrm{p}$-value) $p=.0001$ were calculated. As the calculated $p$-value is lower than .01, the zero hypothesis $H_{0}$ is rejected at the level of significance .01 in favor of the alternative hypothesis. Thus, the test confirmed that different groups of students were statistically significantly different in terms of post-test points.

Considering the fact that the test has confirmed a statistically significant difference between the observed groups, we were interested which groups are statistically significantly different to each other. This question was answered by the Kruskal - Wallis multiple comparison test that was conducted in the STATISTICA program. In the output assembly from the computer, using the Kruskal - Wallis multiple comparison test, the Z - score values and the corresponding $\mathrm{p}$-values were obtained. Because the test hypothesis $H_{0}$ is rejected at the level of significance .05 or .01 , if the calculated $\mathrm{p}$-value is low $(\mathrm{p}<.05$ or $\mathrm{p}<.01)$ and $H_{0}$ cannot be rejected, if the calculated p-value $p .05$, only the table with calculated $\mathrm{p}$ - values is shown from the output assembly (Table11).

Table11. Results of the Kruskal-Wallis multiple comparison test in post-test.

\begin{tabular}{lccc}
\hline & EG & CG1 & CG2 \\
\hline EG & $.0007^{*}$ & $0^{*}$ \\
\hline CG1 & & 1.0000 \\
\hline
\end{tabular}

Table 11 shows that based on the post-test results, the experimental group (EG) differs statistically significantly from both control groups VG1 and CG2. However, the differences in post-test results between control groups CG1 and CG2 are not statistically significant. The situation is illustrated in Figure 2.

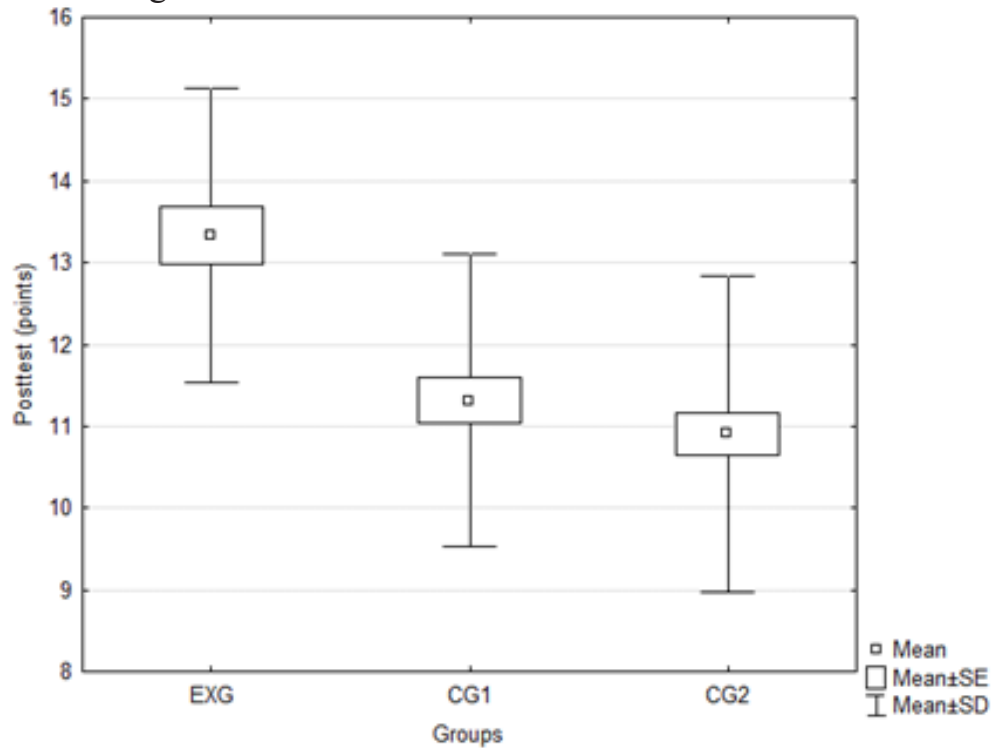

Figure 2. Post-test results (average number of points in each group).

Based on the results obtained by using the Kruskal-Wallis test and also on the calculated average number of points in the post-test, it can be concluded that the EG students achieved in the post-test statistically significantly better results than the students in both control groups (CG1 and CG2). This means that the use of the new teaching method was effective. 
Dalibor GONDA, Anna TIRPAKOVA. A new teaching method aimed at eliminating the causes of students' unsuccessful algorithmic problem solving with parameter

Comparing the results of the pre-test with the post-test results in each group of students (CG1, CG2 and EG), it can be seen that changes occurred in all groups - the results have improved. There was a question arisen whether this improvement was also statistically significant in each group. For illustration, the table is presented (Table 12) with the pre-test and post-test results in each group of students.

Table12. Pre-test and post-test results in three groups of respondents (average number of points).

\begin{tabular}{ccccccccc}
\hline & \multicolumn{2}{c}{ Task 1 } & \multicolumn{2}{c}{ Task 2 } & \multicolumn{2}{c}{ Task 3 } & \multicolumn{2}{c}{ Task 4 } \\
\hline & Pre-test & Post-test & Pre-test & Post-test & Pre-test & Post-test & Pre-test & Post-test \\
\hline CG1 & 3.98 & 3.88 & 4.00 & 3.22 & .98 & 2.41 & 1.66 & 1.80 \\
\hline CG2 & 3.93 & 3.91 & 4.00 & 3.13 & .96 & 2.13 & 1.57 & 1.75 \\
\hline EG & 3.74 & 3.85 & 4.00 & 3.37 & .96 & 2.93 & 1.93 & 3.19 \\
\hline
\end{tabular}

Since the assumption of a normal distribution of the observed signs is not warranted, to verify the statistical significance of the differences between the results obtained by the students in the pre-test and post-test in each group, a nonparametric One Side Wilcoxon Signed Rank Test was used, that represents a nonparametric analogy of the pair parametric t-test. The observed characters will be represented by $\mathrm{X}, \mathrm{Y}$, where $\mathrm{X}$ is the number of points achieved in the EG students' test before the beginning of the experiment, and $\mathrm{Y}$ is the number of the EG students' points in the post-test after finishing of experiment. The values of the $\mathrm{X}$ sign are therefore the results of the EG students in the pre-test and the values of the Y sign are the results of the EG students in the post-test.

The hypothesis $H_{0}$ was tested: medians of the signs $X, Y$ are equal to the one-sided alternative hypothesis $H_{1}$. The test in the STATISTICA program was realized. After inserting the input data, the following results in the output assembly of the computer were obtained: value of the test criteria from the One side Wilcoxon Signed Rank test $(Z=3.9770)$ and $\mathrm{p}$-value $(p=$ .0001).

The test using the p-value was evaluated. Because the calculated p-value is a small number, is rejected at the level of significance This means that the results of the mathematical test were significantly improved in the EG students' group by using a new approach to teaching mathematics. Analogically, by the One side Wilcoxon Signed Rank Test, the results obtained by the students of CG1 (resp. CG2) groups in the pre-test and the post-test were compared. Resulting values of the $\mathrm{Z}$-test criterion and p-values are listed in the table (Table 13).

Table 13. Wilcoxon Single-Selection Test results.

\begin{tabular}{lll}
\hline Groups & $\boldsymbol{Z}$ & $\boldsymbol{p}$-level \\
\hline EG & 3.9770 & .0001 \\
\hline CG1 & 1.6732 & .0943 \\
\hline CG2 & 1.2115 & .2257 \\
\hline
\end{tabular}

Based on the results shown in Table 13, it can be seen that the p-value is lower only in the case of the experimental group. For the control groups CG1 and CG2 the p-value greater than 0.05 was obtained. This means that differences in the pre-test and post-test results in the students' groups CG1, resp. CG2 are not statistically significant, respectively it can be concluded 
Dalibor GONDA, Anna TIRPAKOVA. A new teaching method aimed at eliminating the causes of students' unsuccessful algorithmic problem solving with parameter

OF EDUC

IN THE $21^{\text {st }}$ CENTURY Vol. 76, No. 4, 2018

516

that improving the knowledge of the selected part of mathematics in CG1 and CG2 groups, which occurred during the lesson, is not statistically significant. (= CG1 and CG2 students improved themselves in solving tasks with parameter but not significantly). Based on the above results can be stated that the experiment confirmed the effectiveness of the new approach to teaching the stated subject.

\section{Discussion}

Table 14 shows how the numbers of students who obtained the appropriate number of points for Task 3 and Task 4 solutions both in the pre-test and post-test in each group have been changed. Considering that there were not the same number of students in the groups, the percentages were used.

Table 14. Number of students (in\%) who obtained the appropriate number of points for Task 3 and Task 4 solutions in the pre-test and post-test.

\begin{tabular}{|c|c|c|c|c|c|c|c|c|c|c|c|}
\hline \multirow{3}{*}{ Groups } & \multirow{3}{*}{ Tasks } & \multicolumn{10}{|c|}{ Points } \\
\hline & & \multicolumn{2}{|c|}{0} & \multicolumn{2}{|c|}{1} & \multicolumn{2}{|c|}{2} & \multicolumn{2}{|c|}{3} & \multicolumn{2}{|c|}{4} \\
\hline & & $\begin{array}{l}\text { Pre- } \\
\text { test }\end{array}$ & $\begin{array}{c}\text { Post- } \\
\text { test }\end{array}$ & $\begin{array}{l}\text { Pre- } \\
\text { test }\end{array}$ & $\begin{array}{c}\text { Post- } \\
\text { test }\end{array}$ & $\begin{array}{l}\text { Pre- } \\
\text { test }\end{array}$ & $\begin{array}{c}\text { Post- } \\
\text { test }\end{array}$ & $\begin{array}{l}\text { Pre- } \\
\text { test }\end{array}$ & $\begin{array}{c}\text { Post- } \\
\text { test }\end{array}$ & $\begin{array}{l}\text { Pre- } \\
\text { test }\end{array}$ & $\begin{array}{c}\text { Post- } \\
\text { test }\end{array}$ \\
\hline \multirow{2}{*}{ EG } & 3 & 40.7 & .0 & 33.3 & 11.1 & 18.5 & 25.9 & 3.7 & 22.2 & 3.7 & 40.7 \\
\hline & 4 & 25.9 & 3.7 & 14.8 & 7.4 & 25.9 & 7.4 & 7.4 & 29.6 & 25.9 & 51.9 \\
\hline \multirow{2}{*}{ CG1 } & 3 & 34.1 & .0 & 41.5 & 17.1 & 19.5 & 46.3 & 2.4 & 14.6 & 2.4 & 22.0 \\
\hline & 4 & 19.5 & 22.0 & 41.5 & 24.4 & 14.6 & 14.6 & 2.4 & 29.3 & 22.0 & 9.8 \\
\hline \multirow{2}{*}{ CG2 } & 3 & 37.5 & 3.6 & 39.3 & 19.6 & 16.1 & 48.2 & 3.6 & 17.9 & 3.6 & 10.7 \\
\hline & 4 & 25.0 & 19.6 & 35.7 & 28.6 & 16.1 & 17.9 & 3.6 & 25.0 & 19.6 & 8.9 \\
\hline
\end{tabular}

From Table 14 it can be seen that up to $41 \%$ of students obtained a full score ( 4 points) in the post-test. In CG1 a full score was obtained by $22 \%$ of students, and in CG 2 only $11 \%$ of students obtained this score. This means that the students in CG1 and CG2 groups, who took the traditional course, also had post-test problems with the solution of inequalities with parameter. The results from Task 4 solution in the post-test can also be interpreted.

The tasks in the post-test were chosen on purpose - in order to find out the students' shift in acquiring the term parameter and in working with the conditions of the various teaching methods. The main criterion for choosing Task 3 and Task 4 was that their algorithmic solution should not be the easiest way to obtain results. The post-test result showed a significant EG group shift from an algorithmic approach to creative search for a solution. In both CG1 and CG2 groups this shift was not detected.

The aim of the Task 3 was to find out to what extent students incorporate the specified conditions of solvability into the solution process. In the control groups, it was also recorded in $68 \%$ of students the solution at the interval $(0 ; 1)$ even though, under specified conditions, the solution from this interval is not acceptable. In EG group this solution was recorded in 12\% of students. At the same time, in the control groups they "forgot" to make intersection in $34 \%$. In the experimental group the percentage was only $3 \%$, which indicates the determination of conditions in the control groups to a great extent as part of the algorithm. Algorithmic work with conditions contributed to a lower control groups' success rate also in other post-test tasks. 
Task 3 was focused on understanding the term parameter. Understanding the term $p a$ rameter and its perception was reflected in the process of the task solution. In the EG group there was predominantly used the process described above - where the parameter was perceived as a set of numbers. However, control groups were dominated by the following procedure: First, they canceled the right side $\frac{x-3}{a}-10>0$ and then created a fraction on the left side $\frac{x-3-10 a}{>0}>0$. Zero points are $=0 ; x=10 a+3$. Then both zero points were shown on the same numerical axis and the zero point was considered positive. This solution appeared in $73 \%$ of solutions in the control groups (CG), and it strongly contributed to a significantly lower success rate of the CG in solving this task. In comparison, this solution occurred in $29 \%$ of all solutions in the experimental group (EG). Another remarkable fact is that in $81 \%$ of the EG students there was a change in the solving procedure (procedure stated when describing the post-test) but in the CG it happened only in $34 \%$ of the cases. It can be summarized that EG was largely creative. Creativity was also evident in the ability to find a new approach to the task solution if the originally chosen one was proved to be inappropriate. In the control groups, the algorithm was automatically applied: "Letter in denominator = inequality with unknown variable in the denominator" and the subsequent assignment of the learned algorithm of solution. If the algorithm "failed", the task is "insolvable".

The aim of Task 4 was to find out to what extent the students are able to work with the term parameter in tasks of an unknown type (neither logarithmic inequalities nor equations with parameter were taught in any of groups). It turned out that in EG this task was not solved by only $.03 \%$ of the students, while in the control groups $21 \%$ of the students did not solve the task. It turned out that the missing algorithm in the CG prevented these students from solving the task. The rest of students in the GC used an algorithm to solve the logarithmic inequality. They did not set the solution conditions that would show them the inappropriateness of this algorithm. Their solution looked as follows: Based on the basic sentence about logarithms, it is possible to start solving the inequality $\frac{x-2}{a x}>10$. After canceling the right side and adjusting the left side to one fraction, the inequality $\frac{x(1-10 a)-2}{a x}>0$ was solved. None of the CG students was able to continue in the solution process. Only 13\% of them were looking for another solution. In EG, in $71 \%$ of cases, it has been identified, as the first step in the solution, the effort to set solution conditions, i.e. to solve the inequality $\frac{x-2}{a x}>0$. The difficulty of solving this inequality was for up to $89 \%$ of students an indicator of looking for other than an algorithmic solution of the task. The significant difference in the tasks solution success rate between EG and CG can be basically seen in the cumulating of the inadequacy of an algorithmic approach to the task solution.

In the first phase of our research, in particular, it was shown that our respondents, graduates of various secondary schools, were taught in the form of handing over the final solutions of the types of tasks mainly in the form of algorithms. Navarro-Ibarra (2017) has also come to the same results. At the same time, in the first stage of research, it was found that our respondents, even they decided to devote to mathematics in their professional lives, have learned mathematics mainly on the basis of taking over the solutions. This also resulted in low success in solving problems with a parameter in pre-test that were selected so that they could not be solved using the algorithm alone. Under these circumstances, it can be assumed that they will also teach mathematics in the same way during their pedagogical activities. According to (Escalera-Chávez et al 2017, Boaler, 2016), such a way of teaching mathematics leads to the situation, when joy and enthusiasm of children in mathematics are replaced by fear and disgust from school mathematics in the form of methods, lessons and algorithms that must be learned 
Dalibor GONDA, Anna TIRPAKOVA. A new teaching method aimed at eliminating the causes of students' unsuccessful algorithmic problem solving with parameter

$\begin{array}{r}\text { PROBLEMS } \\ \text { OF EDUCATION } \\ \text { IN THE 21 }{ }^{\text {st }} \text { CENTURY } \\ \text { Vol. 76, No. 4, } 2018 \\ \hline 518\end{array}$

ing

to remember. The new method of teaching the term "parameter" means a deflection of the teaching mathematics from the teaching algorithms to focusing on understanding the new concept and its creative use in solving various problems. This deflection offers learners, among other things, the opportunity to demonstrate their creativity as well. According to Devlin's research (2006), the use of mathematics and mathematical thinking is a natural feature of human being, and it is therefore appropriate to create methods of teaching mathematics aimed at developing that person's feature.

In the second stage of our pedagogical research, it was confirmed that kids in EG were able to solve, in contrast to CG learners, also unknown and creative tasks, in which the concept of the parameter taught by the presented method appeared.

\section{Conclusions}

Based on the presented pedagogical research, it was confirmed that teaching focused on the learning algorithms, which is basically a teaching aimed at achieving results, is limiting for students. It limits their solving skills to solving familiar tasks. Teaching aimed at understanding the concept and its integration into the world of thinking - teaching aimed at solving skills enhances the mathematical solving erudition of students. They are taught to find their own solutions with the creative use of their own knowledge. The effort was to show that there is another way to teach mathematics. And that's a way where the students are shown that mathematics is not just about rigid rules and algorithms, but these rules are the result of creativity and imaginativeness in finding solutions to given tasks. The research also confirmed that this path is also more effective for students.

It is believed that learners are more motivated if they deal with diverse tasks where they naturally follow the already acquired knowledge and spread new insights to find new ways to solve problems. Such a way of acquiring a new concept or new knowledge is a challenge for them to reveal the mystery of the new. The tasks with a parameter provide space for creative search of their own task solving procedures. Their great benefit is also that these tasks help students to use the already acquired knowledge and skills in various variations and combinations to develop creativity and enhance self-efficacy. In the end, learners learn "mathematical self-reliance". This means that the learner can solve the tasks based on his own knowledge and skills in his / her own way. The essential thing when solving this type of tasks becomes what you know and not just if he/she remembers a universal solving procedure. On the basis of this internal motivation factor, the way of kids learning is changing.

\section{References}

Abdullah, A. H. (2016). Mathematics teachers' level of knowledge and practice on the implementation of higher-order thinking skills (HOTS). EURASIA Journal of Mathematics, Science and Technology Education, 13, 3-17. doi:10.12973/eurasia.2017.00601a.

Bardini, C., Radford, L., \& Sabena, C. (2005). Struggling with variables, parameters, and indeterminate objects or how to go insane in mathematics. In Chick, H. L. \& Vincent, J. L. (Eds.), Proceedings of the 29th conference of the international group for the psychology of mathematics education (Vol. 2, pp. 129-136). Melbourne: PME.

Bednarz, N., Kieran, C., \& Lee, L. (1996). Approaches to algebra: Perspectives for research and teaching. Approaches to Algebra, 3-12. doi:10.1007/978-94-009-1732-3_1.

Boaler, J. (2016). Mathematical mindsets. Jossey-Bass, A Wiley Brand, San Francisco.

Devlin, K. (2006). The math instinct: Why you're a mathematical genius (along with lobsters, bird, cast, and dogs). Basic Books: New York.

Escalera-Chávez, M. E. (2016). Factors that promote anxiety toward math on high school students. EURASIA Journal of Mathematics, Science and Technology Education, 13 (1). doi:10.12973/ eurasia.2017.00611a. 
Dalibor GONDA, Anna TIRPAKOVA. A new teaching method aimed at eliminating the causes of students' unsuccessful algorithmic problem solving with parameter

Hoffman, B. (2010). „I think I can, but I'm afraid to try“: The role of self-efficacy beliefs and mathematics anxiety in mathematics problem-solving efficiency. Learning and Individual Differences, 20 (3), 276-283. doi: 10.1016/j.lindif.2010.02.001.

Kubiatko, M., Torkar, G., \& Rovnanova, L. (2017). The teacher as one of the factors influencing students' perception of biology as a school subject. CEPS Journal, 7 (2), 127-140.

Ma, L. (1999). Knowing and teaching elementary mathematics: Teachers' understanding of fundamental mathematics in China and the United States. LEAP, Mahwah - NewJersey.

MacGregor, M., \& Stacey, K. (1993). Seeing a pattern and writing a rule. In I. Hirabayashi, N. Nohda, K. Shigematsu \& F. Lin (Eds), Proceedings of the 17th International conference for the Psychology of Mathematics Education (Vol. 1, pp. 181- 188). University of Tsukuba, Japan. https://doi. org/10.2307/749345.

Kramarski, B., Mevarech, Z. R., \& Arami, M. (2002). The effects of metacognitive instruction on solving mathematical authentic tasks. Educational Studies in Mathematics, 49 (2), 225-250. doi:10.1023/a:1016282811724.

Navarro-Ibarra, L., García-Santillán, A., Cuevas-Salazar, O., \& Ansaldo-Leyva, J. (2017). Mathematics, technology and learning: How to align these variables in order to explain anxiety towards mathematics and attitude towards the use of technology for learning mathematics. Eurasia Journal of Mathematics, Science and Technology Education, 13 (9), 6211-6229. doi:10.12973/ eurasia.2017.01060a.

Petty, G. (1996). Moderní Vyučování [Modern teaching]. Potrál Praha.

Radford, L. (1996). The roles of geometry and arithmetic in the development of elementary algebra. In N. Bednarz et al. (Eds.), Approaches to Algebra: Perspectives for research and teaching (p. 39-53). Dordrecht: Kluwer. https://doi.org/10.1007/978-94-009-1732-3 3.

Schoenfeld, A., \& Arcavi, A. (1988). On the meaning of variable. Mathematics Teacher, 81 (6), 420-427.

Sierpinska, A. (1994). Understanding in mathematics. The Falmer Press, London-Bristol. https://doi. org/10.4324/9780203454183.

Supekar, K., Swigart, A. G., Tenison, C., Jolles, D. D., Rosenberg-Lee, M., Fuchs, L., Menon, V. (2013). Neutral predictors of individual differences in response to math tutoring in primary-grade school children. Proceedings of the National Academy of Sciences, 110 (20), 8230 - 8235.

Trigueros, M., \& Ursini, S. (1999). Does the understanding of variable evolve through schooling? In O. Zaslavsky (Ed.), Proceedings of the 23rd conference of the international group for the psychology of mathematics education (Vol. 4, pp. 273-280). Haifa, Israel: PME.

Wolfram, C. (2010). Teaching kids real math with computers. TED Talks. http://ted.com/talks/conrad wolfram_teaching_kids_real_math_with_computers?lanfuage=en.

Znám, Š., Bukovský, L., Hejný, M., Hvorecký, J., \& Riečan, B. (1986). Pohl’ad do dejín matematiky [View into the history of mathematics]. Alfa: Bratislava.

Received: April 09, 2018

Accepted: August 02, 2018

\begin{tabular}{l} 
OF EDUCATION \\
IN THE 21 $1^{\text {st }}$ CENTURY \\
Vol. 76, No. 4, 2018 \\
\hline 519
\end{tabular} 